\title{
A CANTOR-LEBESGUE THEOREM IN TWO DIMENSIONS ${ }^{1}$
}

\author{
ROGER COOKE
}

\begin{abstract}
We prove a partial extension of the classical result of Cantor and Lebesgue to two dimensions, for circular summation of trigonometric series. We assume convergence almost everywhere rather than merely on a set of positive measure. Although the theorem we prove has applications in uniqueness theorems, its purpose is to suggest what may be true in general, since the proof does not generalize to higher dimensions.
\end{abstract}

The purpose of this paper is to prove a theorem which partly extends the well-known Cantor-Lebesgue theorem. To state the theorem we introduce the following notation: $\sum_{m} c_{m} \exp [i(m, x)]$ denotes a trigonometric series in $k$ variables, $m=\left(m_{1}, m_{2}, \cdots, m_{k}\right)$ is a lattice point in $k$-dimensional space, $x=\left(x_{1}, x_{2}, \cdots, x_{k}\right)$ is a point of $k$-dimensional space, $(m, x)=m_{1} x_{1}+\cdots+m_{k} x_{k}, \quad|m|^{2}=(m, m)$, $A_{n}(x)=\sum_{|m|^{2}=n} c_{m} \exp [i(m, x)], \quad\|f\|_{1}=(2 \pi)^{-k} \int T^{k}|f(x)| d x,\|f\|_{2}$ $=\left[(2 \pi)^{-k} \int_{T^{k}}|f(x)|^{2} d x\right]^{1 / 2}$, and $T^{k}$ is the torus with Lebesgue measure $d x$. We use the now standard $k$-dimensional notation even though we are principally interested in the case $k=2$.

TheOREM. Let $k=2$. If $A_{n}(x)$ tends to 0 almost everywhere as $n$ tends to infinity, then $\left\|A_{n}\right\|_{2}^{2}=\sum_{|m|^{2}=n}\left|c_{m}\right|^{2}$ tends to 0 also.

The theorem presents interest for two reasons. First, as Paul Cohen has shown in his dissertation at the University of Chicago, the analogue of the Cantor-Lebesgue theorem is false for square summation. Second, many of the results on uniqueness of multiple trigonometric series have had to make use of some rather strong assumptions, for instance the uniform convergence of the series $\sum_{n=1}^{\infty} n^{-1} A_{n}(x)$, cf. [1]. It is hoped that this result will be only one of many similar ones. If it can be extended or strengthened, many of these strong assumptions could be dispensed with. As an example, we

Presented to the Society, October 10,1970 under the title A Cantor-Lebesgue theorem for two dimensions; received by the editors July 27, 1970.

AMS 1970 subject classifications. Primary 42A48, 42A92; Secondary 10E30.

Key words and phrases. Cantor-Lebesgue theorem, spherical summation, uniqueness of trigonometric series, multiple trigonometric series, lattice points in Euclidean space.

1 This work was supported by NSF grant GP-12882. 
combine our result with a much deeper result of Shapiro to obtain the following.

Corollary. Let $k=2$. If $\sum_{n=0}^{\infty} A_{n}(x)=0$ for $x \neq 2 \pi m$, then $c_{m}=0$ for all $m$.

The proof of the theorem requires two lemmas.

Leмма 1. Let $(X, \mu)$ be a finite measure space, and let $f_{n}(x)$ be a sequence of nonnegative measurable functions on $X$ such that $f_{n}(x)$ tends to 0 almost everywhere and the indefinite integrals $\int f_{n} d \mu$ are uniformly absolutely continuous. Then $\int_{X} f_{n} d \mu$ tends to 0 .

Proof. Let $\epsilon>0$. Choose $\delta>0$ such that $\int_{E} f_{n} d \mu<\epsilon$ for all $n$ if $\mu(E)<\delta$. Since $f_{n}(x)$ tends to 0 in measure, choose $N$ so large that $\mu\left(\left\{x: f_{n}(x)>\epsilon\right\}\right)<\delta$ when $n>N$. Then if $n>N$, we have

$$
\int_{X} f_{n} d \mu=\int_{f_{n} \leqq \epsilon} f_{n} d \mu+\int_{f_{n}>\epsilon} f_{n} d \mu<\epsilon+\epsilon \cdot \mu(X) \text {. Q.E.D. }
$$

Similar lemmas are common in measure theory, cf. [2, pp. 100-101].

Lemma 2. Let $(X, \mu)$ and $f_{n}$ be as above. If there exists $M$ such that $\int_{X} f_{n}^{2} d \mu<M$ for all $n$, then the indefinite integrals $\int f_{n} d \mu$ are uniformly absolutely continuous.

Proof. Let $N$ be any positive number, and write $f_{n}=g_{n}+h_{n}$, where $g_{n}=f_{n}$ if $f_{n} \leqq N$ and $g_{n}=0$ on the set where $f_{n}>N$. Then $\int_{E} f_{n} d \mu=\int_{E} g_{n} d \mu+\int_{E} h_{n} d \mu \leqq N \cdot \mu(E)+N^{-1} \int_{E} f_{n}^{2} d \mu$.

The inequality results from the fact that $f_{n}^{2} \geqq N h_{n}$. Thus $\int_{E} f_{n} d \mu$ $\leqq N \cdot \mu(E)+M N^{-1}$ : Given $\epsilon>0$, choose $N$ so that $M N^{-1}<\epsilon$; then if $\mu(E)<\epsilon N^{-1}$, we have $\int_{E} f_{n} d \mu<2 \epsilon$. Q.E.D.

This lemma is essentially the result on p. 143 of [4].

Proof of the Theorem. We have

$$
\left|A_{n}(x)\right|^{2}=\sum_{|m|^{2}=|p|^{2}=n} c_{m} \bar{c}_{p} \exp [i(m-p, x)]=\sum_{q} \sum c_{m} \bar{c}_{p} \exp [i(q, x)]
$$

where the inner sum is extended over all pairs of lattice points $m$ and $p$ such that $|m|^{2}=|p|^{2}=n$ and $m-p=q$. Now it is easily seen that when $k=2$ this inner sum contains at most two terms. For, given a chord on a circle, there is at most one other chord on the circle having the same length and parallel to it. Hence if $B_{n}(x)=\left|A_{n}(x)\right|^{2}$, Parseval's identity implies that

$$
\left\|B_{n}\right\|_{2}^{2}=\sum_{q}\left|\sum c_{m} \bar{c}_{p}\right|^{2}
$$


Thus

$$
\begin{aligned}
\left\|B_{n}\right\|_{2}^{2} & \leqq\left\|A_{n}\right\|_{2}^{4}+2 \sum_{q \neq 0} \sum_{\mid \neq 0}\left|c_{m} \bar{c}_{p}\right|^{2} \\
& \leqq\left\|A_{n}\right\|_{2}^{4}+2 \sum_{|m|^{2}=|p|^{2}=n}\left|c_{m}\right|^{2}\left|c_{p}\right|^{2} \\
& =2\left[\sum_{|m|^{2}=n}\left|c_{m}\right|^{2}\right]^{2}+\left\|A_{n}\right\|_{2}^{4}=3\left\|A_{n}\right\|_{2}^{4} .
\end{aligned}
$$

It now follows from Lemma 2 that if the sequence $\left\|A_{n}\right\|_{2}$ is bounded, then the integrals $\int\left|A_{n}(x)\right|^{2} d x$ are uniformly absolutely continuous; hence in this case $\int_{T^{2}}\left|A_{n}(x)\right|^{2} d x$ tends to 0 by Lemma 1 . In the general case we replace $A_{n}(x)$ by $C_{n}(x)=\left\|A_{n}\right\|_{2}^{-1} A_{n}(x)$. If $\left\|A_{n}\right\|_{2}$ does not tend to 0 , there is a subsequence of the positive integers, $n_{k}$, such that $\left\|A_{n_{k}}\right\|_{2} \geqq c>0$. Then by what has already been shown, $\left\|C_{n_{k}}\right\|_{2}$ tends to 0 . But this is absurd, since $\left\|C_{n_{k}}\right\|_{2}=1$. This proves the theorem.

The proof given here breaks down for square partial sums at inequality (1). (The number of terms in the inner sum does not remain bounded.) For spherical sums in dimensions higher than 2 the proof would carry over if it could be shown that the number of pairs of lattice points $m$ and $p$, on a given sphere and such that $m-p=q$, remains bounded (over all spheres). That such is not the case, however, is easily seen. Given a lattice point in 2 -space, $\left(r_{1}, r_{2}\right)$, there is a pair $m=\left(r_{1}, r_{2},+1\right), p=\left(r_{1}, r_{2},-1\right)$ on the sphere of radius $\left(r_{1}^{2}+r_{2}^{2}+1\right)^{1 / 2}$ in 3 -space with $m-p=(0,0,2)$. The question now becomes whether the number of lattice points on the circle of radius $r$ with center at $(0,0)$ is a bounded function of $r$. This function is not bounded, as one can easily deduce. From number theory it is known that the number of lattice points on the circle of radius $\sqrt{ } r$ about $(0,0)$ is four times the difference between the number of divisors of $r$ of the form $4 n+1$ and the number of divisors of the form $4 n+3$. Thus the circle about $(0,0)$ of radius $5^{r}$ contains $8 r+4$ lattice points, from which the unboundedness of the function follows immediately.

ProOF OF THE CoRollary.

$$
\sum_{|m|^{2}=n}\left|c_{m}\right| \leqq\left(\sum_{|m|^{2}=n}\left|c_{m}\right|^{2}\right)^{1 / 2}\left(\sum_{|m|^{2}=n} 1\right)^{1 / 2}=o(1) \sqrt{ } N_{n},
$$

where $N_{n}$ denotes the number of lattice points on the sphere of radius $\sqrt{ } n$. Hence we have 


$$
\begin{aligned}
\sum_{R-1 \leqslant|m|<R}\left|c_{m}\right| & =o(1)\left(\sum_{(R-1)^{2} \leq n<R^{2}} \sqrt{ } N_{n}\right) \\
& \leqq o(1)\left(\sum_{(R-1)^{2} \leq n<R^{2}} N_{n}\right)^{1 / 2}(2 R-1)^{1 / 2} \\
& =o(1) O\left(R^{1 / 2}\right) O\left(R^{1 / 2}\right)=o(R)
\end{aligned}
$$

Here we have used the Schwarz inequality twice to obtain inequalities (2) and (3). To obtain (4) we have used the fact that $\sum_{(R-1)^{2} \leq n<R^{2}} N_{n}$ is the number of lattice points inside the circle of radius $R$ but not inside the circle of radius $R-1$. It is well known that this number is $O(R)$. Thus the hypotheses of Shapiro's theorem [3, Theorem 7] are satisfied, and so $c_{m}=0$ for all $m$.

\section{BIBLIOGRAPHY}

1. Min-Teh Cheng, Uniqueness of multiple trigonometric series, Ann. of Math. (2) 52 (1950), 403-416. MR 12, 174.

2. P. R. Halmos, Measure theory, Van Nostrand, Princeton, N. J., 1950. MR 11, 504 .

3. V. L. Shapiro, Fourier series in several variables, Bull. Amer. Math. Soc. 70 (1964), 48-93. MR 28 \#1448.

4. A. Zygmund, Trigonometric series. Vols. I, II, 2nd ed., reprinted with corrections and some additions, Cambridge Univ. Press, New York, 1968. MR 38 \#4882.

University of Vermont, Burlington, Vermont 05401 